\title{
CMOS image sensor characterization experimental setup
}

Thierry Avignon, Baptiste Perrin, Jean Augereau, Julien Moreau, Lionel Jacubowiez

Thierry Avignon, Baptiste Perrin, Jean Augereau, Julien Moreau, Lionel Jacubowiez, "CMOS image sensor characterization experimental setup," Proc. SPIE 9793, Education and Training in Optics and Photonics: ETOP 2015, 97931K (8 October 2015); doi: 10.1117/12.2223119

Event: Education and Training in Optics and Photonics: ETOP 2015, 2015, Bordeaux, France 


\title{
CMOS Image sensor characterization experimental setup
}

\author{
Thierry Avignon, Baptiste Perrin, Jean Augereau, Julien Moreau, Lionel Jacubowiez \\ Institut d'Optique Graduate School \\ Laboratoire d’Enseignement Expérimental (LEnsE)
}

2 Avenue Augustin Fresnel, 91127 Palaiseau (France)

\begin{abstract}
We demonstrate a very simple experimental setup that allows our students to study and fully characterize an industrial CMOS Image Sensor. In 2014, 95\% of produced cameras are CMOS image sensors (Complementary Metal Oxyd Semiconductor) and only 5\% are still CCD sensors (ChargeCoupled Device).

The main difference between CMOS and CCD is that each CMOS sensor pixel has its own readout circuit (voltage-photoelectron conversion and amplification) directly adjacent to the photosensitive area. CMOS image sensors are not only cheaper, because simpler to manufacture, they have a lower power consumption than CCD sensors. They also allow image processing at the pixel level (zones of interest (ROI), Binning, filtering, etc ...). However, compare to CCD sensor, CMOS sensors often demonstrate a lower dynamic, a larger read-out noise and a larger non uniformity of the spatial response. In overall, it is very important to understand every characteristic of an image sensor and be able to measure it in a simple way.

Our system consist in a small integrating sphere illuminated by a white LED, a standard calibrated photodiode (or a light power meter) and a small monochromator (or several colored LED). Control of the camera parameters, Image acquisition and data processing are achieved with a single Matlab homemade software.
\end{abstract}

Keywords: CCD and CMOS image sensors characterization, dark signal, readout noise, photon noise, pattern response non-uniformity, quantization, binning, ROI

Education and Training in Optics and Photonics: ETOP 2015, edited by Eric Cormier, Laurent Sarger Proc. of SPIE Vol. 9793, 97931K · ( ) 2015 SPIE, IEEE, OSA, ICO · doi: 10.1117/12.2223119 


\section{CMOS sensor datasheet:}

The purpose of the lab is to measure the characteristics of an industrial camera with a CMOS sensor manufactured by the E2V company (1.3 Mpix $5.3 \mu \mathrm{m}$ pixels square). A part of the datasheet is given below.

Capteur EV76C560 Typical electro-optical performance @ $25^{\circ} \mathrm{C}$ and $65^{\circ} \mathrm{C}$, nominal pixel clock

\begin{tabular}{|c|c|c|c|c|}
\hline \multicolumn{2}{|l|}{ Parameter } & Unit & \multicolumn{2}{|c|}{ Typical value } \\
\hline \multirow{7}{*}{$\begin{array}{l}\text { Sensor } \\
\text { characteristics }\end{array}$} & Resolution & pixels & \multicolumn{2}{|c|}{$1280(\mathrm{H}) \times 1024(\mathrm{~V})$} \\
\hline & Image size & $\begin{array}{r}\mathrm{mm} \\
\text { inches }\end{array}$ & \multicolumn{2}{|c|}{$\begin{array}{c}6.9(\mathrm{H}) \times 5.5(\mathrm{~V})-8.7 \text { (diagonal) } \\
\approx 1 / 1.8\end{array}$} \\
\hline & Pixel size (square) & $\mu \mathrm{m}$ & \multicolumn{2}{|c|}{$5.3 \times 5.3$} \\
\hline & Aspect ratio & & \multicolumn{2}{|c|}{$5 / 4$} \\
\hline & Max frame rate & fps & \multicolumn{2}{|c|}{$60 @$ full format } \\
\hline & Pixel rate & Mpixels / s & \multicolumn{2}{|c|}{$90->120$} \\
\hline & Bit depth & bits & \multicolumn{2}{|c|}{10} \\
\hline \multirow{9}{*}{$\begin{array}{c}\text { Pixel } \\
\text { performance }\end{array}$} & & & $@ 25^{\circ} \mathrm{C}$ & $@ 65^{\circ} \mathrm{C}$ \\
\hline & Dynamic range & $\mathrm{dB}$ & $>62$ & $>57$ \\
\hline & Qsat & ke- & \multicolumn{2}{|c|}{12} \\
\hline & SNR Max & $d B$ & 41 & 39 \\
\hline & MTF at Nyquist, $\lambda=550 \mathrm{~nm}$ & $\%$ & \multicolumn{2}{|c|}{50} \\
\hline & Dark signal $^{(1)}$ & $\mathrm{LSB}_{10} / \mathrm{s}$ & 24 & 420 \\
\hline & $\operatorname{DSNU}^{(1)}$ & $\mathrm{LSB}_{10} / \mathrm{s}$ & 6 & 116 \\
\hline & PRNU ${ }^{(2)}$ (RMS) & $\%$ & \multicolumn{2}{|c|}{$<1$} \\
\hline & Responsivity ${ }^{(3)}$ & $\mathrm{LSB}_{10} /($ Lux.s $)$ & \multicolumn{2}{|c|}{6600} \\
\hline \multirow{2}{*}{$\begin{array}{l}\text { Electrical } \\
\text { interface }\end{array}$} & Power supplies & V & \multicolumn{2}{|c|}{$3.3 \& 1.8$} \\
\hline & $\begin{array}{ll}\text { Power consumption: } & \text { Functional } \\
& \text { Standby }\end{array}$ & $\begin{array}{l}\mathrm{mW} \\
\mu \mathrm{W}\end{array}$ & \multicolumn{2}{|c|}{$\begin{array}{c}<200 \mathrm{~mW} \\
180\end{array}$} \\
\hline
\end{tabular}

\footnotetext{
1. Min gain, 10 bits.

2. Measured @ Vsat/2, min gain.

3. $\quad 3200 \mathrm{~K}$, window with AR coating, IR cutoff filter BG38 $2 \mathrm{~mm}$.

4. @ $@ 60 \mathrm{fps}$, full format, with $10 \mathrm{pF}$ on each output.
}

Figure 1 : Datasheet of the CMOS sensor

We first ask to the student to understand the definitions of each characteristic of the datasheet.

For example $\mathrm{Q}_{\text {sat }}$ is the Full Well capacity. It defines the amount of photoelectrons an individual pixel can hold before saturating.

If the sensor is photon noise limited, the SNR max depends only of the full quantum well capacity:

$S N R=\frac{Q_{\text {sat }}}{\sqrt{Q_{\text {sat }}}}=110$ and $20 \log (110)=41 \mathrm{~dB}$ 
From the full well capacity of one pixel, the students can also deduce an approximate value of the conversion factor between numbers of photo-electrons and level of the digital signal.

$$
G=\frac{Q_{\text {sat }}}{\text { Bit depth }}=1200 / 1024=11.7 \mathrm{e}^{-} / A D U
$$

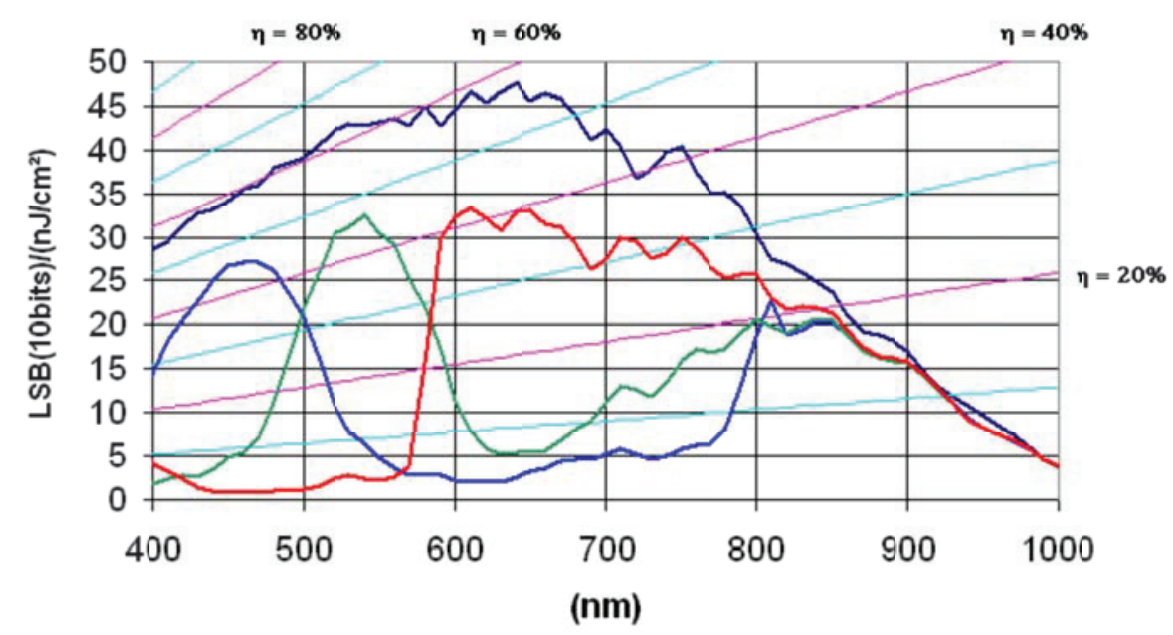

Figure 2 : Spectral response of the sensor (from the datasheet of the sensor)

From this curve, the students can check the consistency between quantum efficiency and the spectral response of the sensor.

For example, at $600 \mathrm{~nm}$, according to this datasheet, the sensor will measure a level of 45 for a radiant exposure of $1 \mathrm{~nJ} / \mathrm{cm}^{\wedge} 2$. This radiant exposure of $1 \mathrm{~nJ}$ correponds to 849 photons. For a quantum efficiency equal to one and conversion factor of 11.7, the sensor would measure a level of 72 (849 / 11.7). A level of 45 given in the datasheet leads to a quantum efficiency: $\eta=45 / 72=0.62$

\section{Experimental set-up:}

The measurements are done with 2 simple experimental setups:

1. For linearity and noise measurement: a small integrating sphere. The source is a white LED driven by an adjustable power supply.

2. For spectral response measurement: a monochromator with a collimator to shine the CMOS sensor with an adjustable wavelength. For each wavelength, a photodiode measure the irradiance of the sensor. 

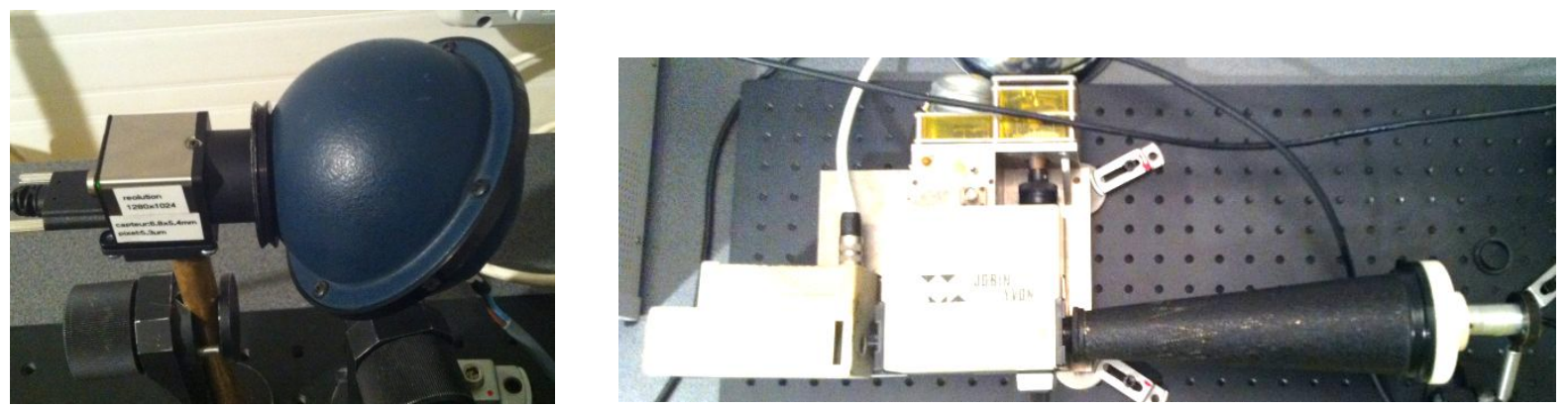

Figure 3 : experimental set-up

\section{Matlab software:}

Matlab homemade software was developed to easily change the parameters of image acquisition and quickly perform measurements of characteristics. An example of measurement is given in figure 4.

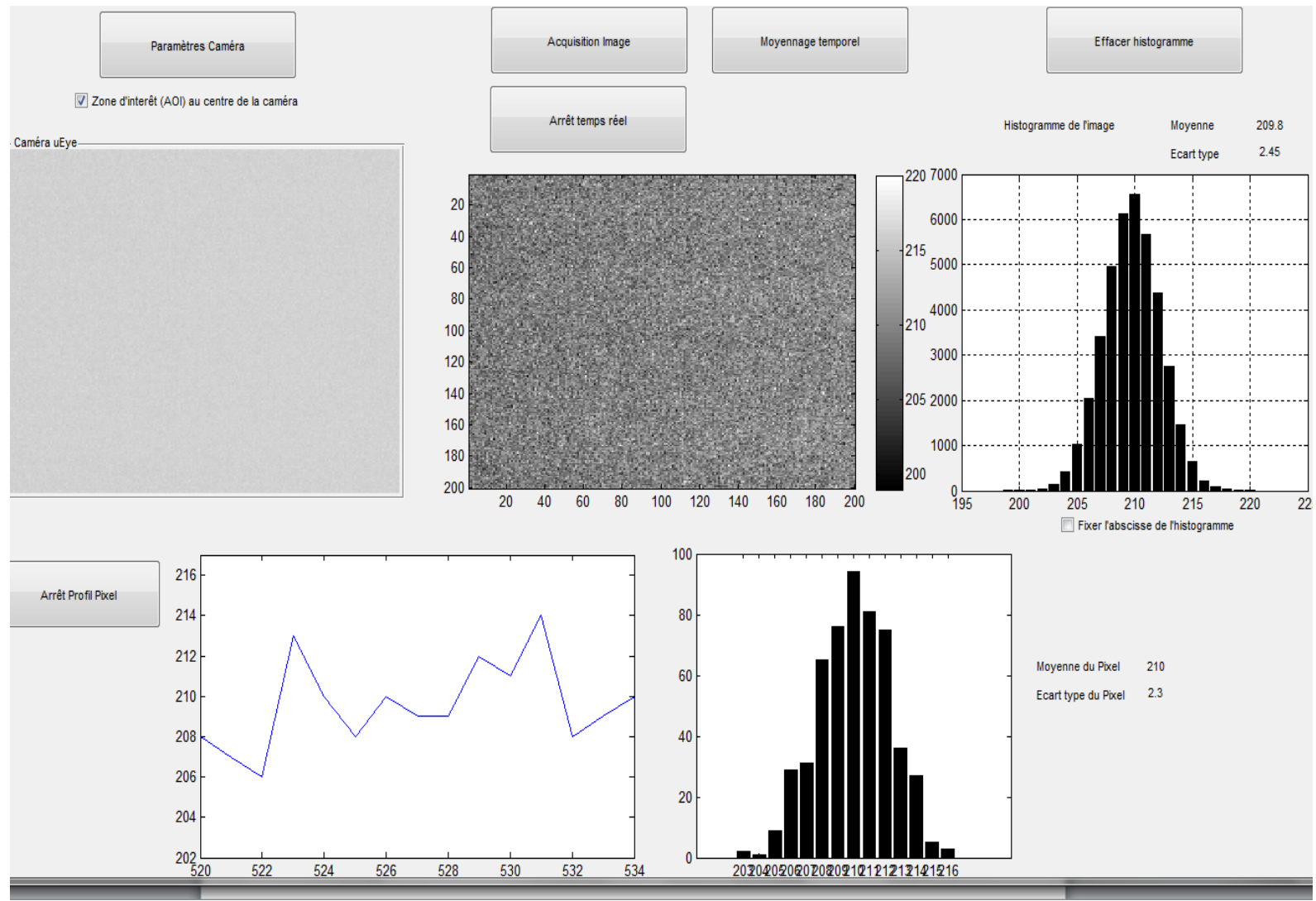

Figure 4 : Home made Matlab GUI

This software allows to choose all the parameters of the sensor and adjust the integration time. Here, we study a region of interest (ROI) of $200 \times 200$ pixels in the middle of the sensor. The 
software dispay the image of the ROI and the corresponding hitogram. We can also follow the value of one pixel and dispay the histogram.

\section{Measurements of the image sensor characteristics:}

1. Readout noise and dark signal:

In the dark with a very small integration time (less than $1 \mathrm{~ms}$ ), we measure a bias of 28 ADU and a the standard type deviation of 1.04 ADU.

Then we increase the integration time from 0 to 2 seconds and measure the mean value on the image. We can see the presence of hot spots on the sensor. For 1 second integration time we find a black level of around $20 \mathrm{ADU} / \mathrm{s}$ to compare with $6 \mathrm{ADU} / \mathrm{s}$ of the datasheet at $25^{\circ} \mathrm{C}$. This value depends dramatically on the temperature of the camera. This explain why, for very low illumination, sensors must be cooled down.

2. Linearity and photon noise:

With the integrating sphere with have an almost uniform irradiance of the sensor. To check the perfect linearity of the sensor with meaure the mean value in the ROI when we increase integration time.

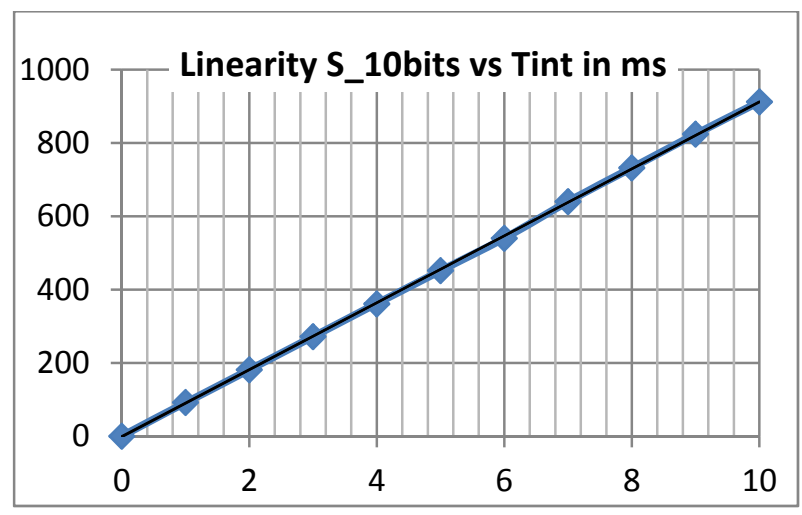

Figure 5 : Sensor linearity

To measure photon noise with measure the fluctuation of the level of one pixel.

Photon noise distribution is a Poisson distribution. The variance of the number of counted photoelectrons in one pixel is equal to the mean value of the number of photo-electrons:

$$
\sigma_{N e}^{2}=<N_{e}>
$$

The number of photoelectrons is converted into a digital signal: $S_{10 b i t s}=N e / G$ where $G$ is the conversion factor or gain.

So the variance of the digital signal should be proportional to the mean value of the digital signal and the slope is the conversion factor of the sensor. Figure 6 is a typical plot that the students will find. 


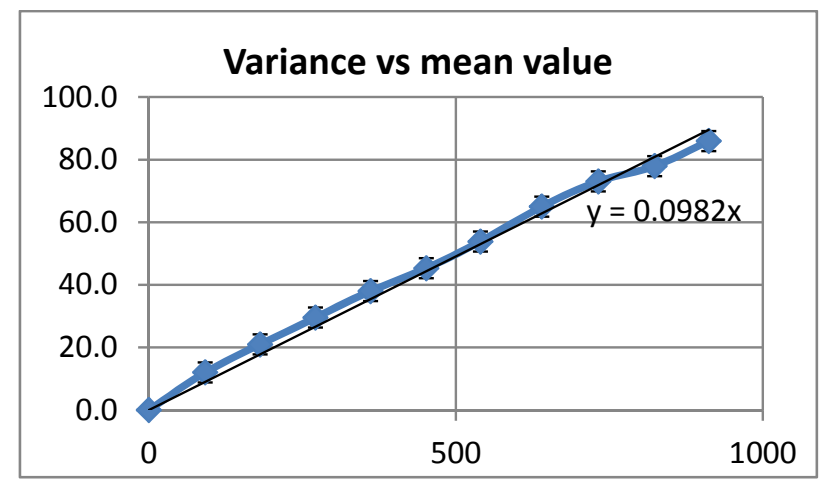

Figure 6 : Photon noise. The variance is proportionnal to the mean value

With this method we measure a gain : $G=10.2+/-0.5 \mathrm{e}^{-} / \mathrm{ADU}$. This value may be compared with $\mathrm{G}=$ 11.7

3. Spectral response of the detector:

For this last measurement, we use a monochomator and photodiode for which we know the spectral response. This photodiode allows measuring the irradianceof the sensor.

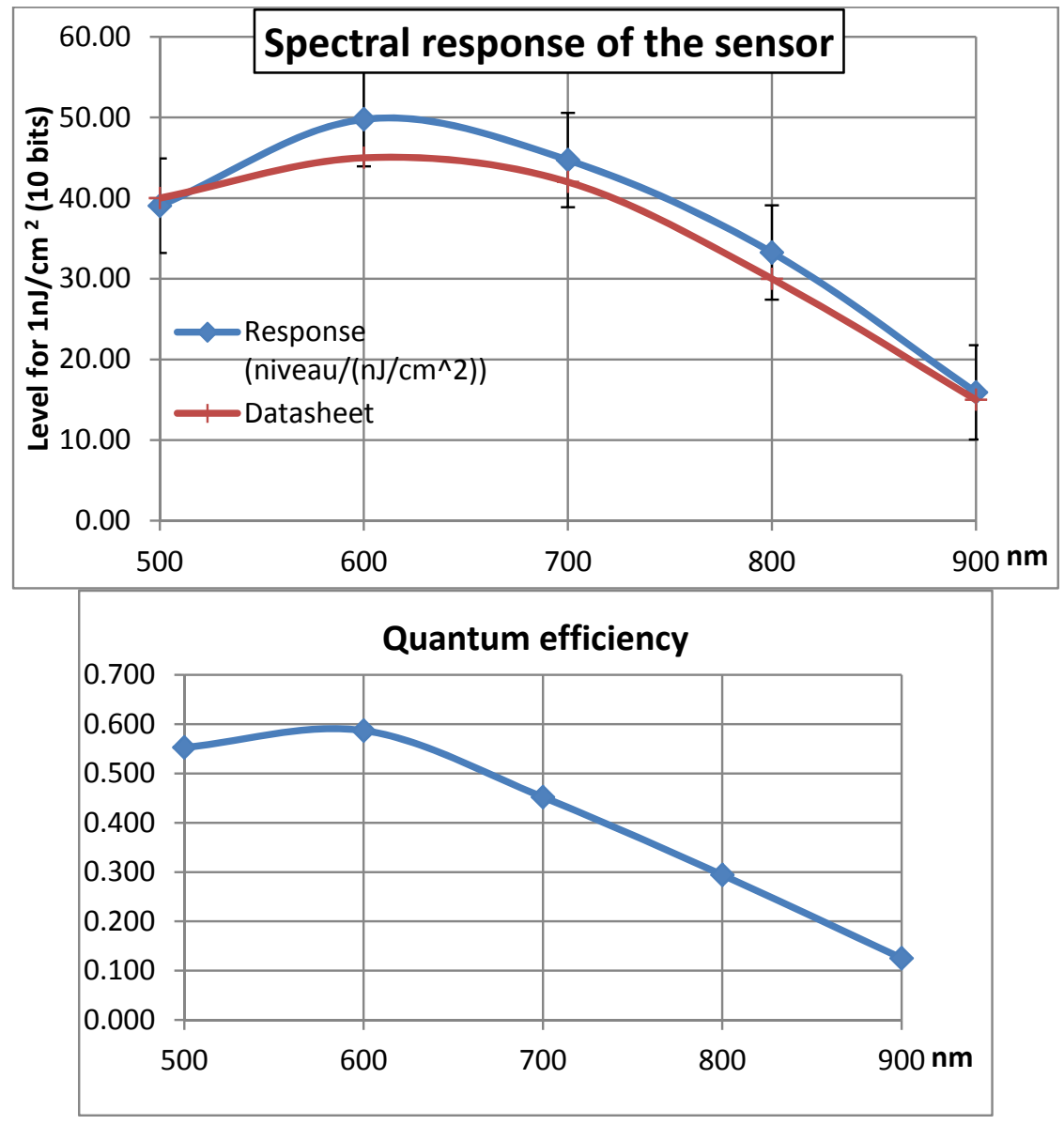

Figure 7 : Typical measurement of the spectral response and quantum efficiency 


\section{Conclusion}

This simple setup allows our undergraduate students to measure and understand the difference between a bias and dark signal. They can measure the readout noise and the nonuniformity of the dark signal. They will see how the dark non-uniformity pattern increases with the integration time. They will check the linearity of the sensor. They will carried out accurate photon noise measurements at the pixel level, and that's seem very important for an future engineer specialized in optics. Finally, they will be able measure the spectral response of the sensor. This simple setup could be used with any image sensor (CMOS or CCD). 